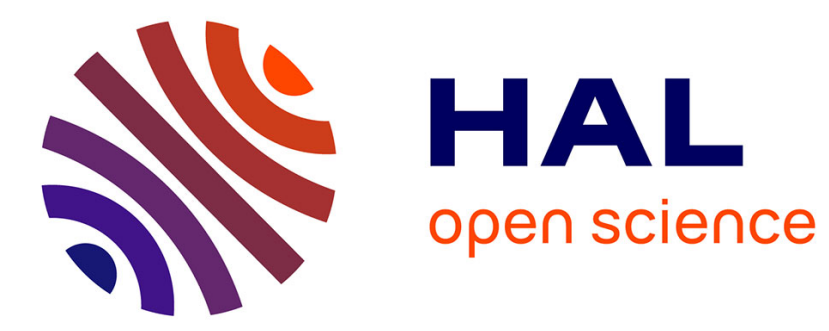

\title{
Towards an industrial deployment of PZT based SHM processes: A dedicated metamodel for Lamb wave propagation
}

\author{
Hadrien Postorino, Marc Rebillat, Eric Monteiro, Nazih Mechbal
}

\section{- To cite this version:}

Hadrien Postorino, Marc Rebillat, Eric Monteiro, Nazih Mechbal. Towards an industrial deployment of PZT based SHM processes: A dedicated metamodel for Lamb wave propagation. European Workshop on Structural Health Monitoring 2020, Jul 2020, Palerme, Italy. pp.1-12. hal-03023307

\section{HAL Id: hal-03023307 https://hal.science/hal-03023307}

Submitted on 25 Nov 2020

HAL is a multi-disciplinary open access archive for the deposit and dissemination of scientific research documents, whether they are published or not. The documents may come from teaching and research institutions in France or abroad, or from public or private research centers.
L'archive ouverte pluridisciplinaire HAL, est destinée au dépôt et à la diffusion de documents scientifiques de niveau recherche, publiés ou non, émanant des établissements d'enseignement et de recherche français ou étrangers, des laboratoires publics ou privés. 


\title{
Towards an industrial deployment of PZT based SHM processes: A dedicated metamodel for Lamb wave propagation
}

\author{
POSTORINO Hadrien ${ }^{1}$, REBILLAT Marc ${ }^{1}$, MONTEIRO Eric ${ }^{1}$, and MECHBAL \\ Nazih $^{1}$ \\ 1. Laboratoire PIMM, Arts et Metiers Institute of Technology, CNRS, Cnam, HESAM \\ Universite, 151 boulevard de l'Hopital, 75013 Paris (France) hadrien. postorino@ensam.eu
}

\begin{abstract}
Numerical simulations of Structural Health Monitoring processes based on wave propagation can be very costly in terms of computation time, especially for complex aeronautic composite structures, and therefore strongly limits the deployment of industrial applications. Metamodels build a relatively simple relationship between inputs and outputs from a set of data and thus can overcome that difficulty. A metamodel based on radial basis functions interpolation is build in order to predict a Lamb Wave measurement on a damaged composite plate equipped by a network of 3 piezoelectric elements. The input parameters describe the position of the damage. This surrogate model is used to predict the measured signals for new damage configurations with a limited computational cost. Moreover, this metamodel is used in a reverse way to solve the inverse problem. A swarm particle optimisation algorithm seeks to find the position of a damage from a set of simulated signals. This approach allows us to identify correctly the damage localisation for an unknown configuration, providing therefore a new method for damage localization.
\end{abstract}

Keywords: SHM · Lamb Waves Propagation · Metamodel · Inverse Problem

\section{Introduction}

In the past decades, composite materials have become omnipresent in the aeronautical, automotive, space and nautical industries [5]. They outperform metallic materials in terms of mechanical and chemical resistance. They allow for the design of lightweight structures with unique mechanical and geometric characteristics. They offer the possibility of reducing the weight of aircraft and increasing their lifespan. However composite structures are also subject to damages caused by fatigue, defect during manufacturing or impact incidents. These damages can typically take the form of cracks, holes, delimitation or discontinuity. Non-destructive testing still 
plays a major role in the industry to ensure the integrity of a structure but needs the interruption of service of the plane for regular human visual inspections.

Thus, the creation of smart structures able to monitor and autonomously determine their structural health has become an important subject of research and innovation over the last decades [3]. These new control and monitoring methods are called Structural Health Monitoring (SHM). SHM has the main objective to determine the structural health in real time. It will then be possible to adapt the maintenance strategy to the actual state of the structure, allowing to optimize the costs and to guarantee more safety.

SHM systems should ideally meet the following requirements [12] : low cost, continuous evaluation, sensitive to small and different types of damage, insensitive to operating conditions, noise and environmental changes. In order to evaluate the maturation of SHM techniques, four levels are distinguished: (i) Damage Detection, (ii) Damage localisation, (iii) Damage quantification and (iv) Damage characterization.

The most advanced techniques in SHM reach level 2 with satisfaction. On the other hand, level 3 and 4 are still active research subjects. These techniques can be grouped into three categories : vibration monitoring, deformation monitoring and wave propagation monitoring. This paper will focus on Lamb wave propagation monitoring.

Lamb waves have the particularity to propagate in plates with low attenuation. When the wave propagates through a damage, it is modified by the damage. By studying and analyzing the wave propagation in the structure, it is then possible to determine the structural state of the structure. The generation and measurement of Lamb waves is done using a network of piezoelectric transducer distributed over the entire structure under study. These PZT transducers act alternately in actuator mode and in sensor mode. The data is then collected and processed to determine the state of the structure. For this purpose, the recorded signals of unknown state are compared with the previously recorded signals of a healthy state. Different values, called Damage Index, allow the comparison of the signals.

The SHM has been subject to a significant amount of works over the past several decades [3]. Applications already exist in civil engineering for the monitoring of buildings, civil structures, roads and railways. In the aeronautics industry, the industrial deployment of research advances has been slow to take place. Various explanations can be given, but one of the main issue is the difficulty to evaluate correctly the performance of SHM systems. The evaluation of SHM systems passed by the computation quantity of interest such as the Probability Of Detection or Probability Of Localisation [13]. This involves to collect a huge amount of data on structures with a lot of damage configurations in order to make statistical evaluation. A fully experimental approach is thus difficult and costly: Moriot [11] robustly eval- 
uated their SHM processes based on experimental data with a plate equipped by piezoelectric transducers.

An evaluation based on numerical data appears to be more feasible. However, to simulate SHM processes is not a trivial task because of the complexity of the aeronautic structures and the large diversity of damage. Numerical simulations can be costly in terms of computational time, but are necessary in order to evaluate the processes and determine their effectiveness.

The creation of a metamodel (or surrogate model) able to predict the effect of a damage on a structure would considerably reduce the computation time and allow to test SHM processes on a large number of damage configurations. Building a metamodel means looking for a relatively simple relationship between inputs and outputs. Many methods of metamodelisation exists for a very large variety of applications. In the context of Lamb waves SHM, Borate et al. [2] recently used Proper Orthognal Decomposition and Artificial Neural Network in order to predict the position of a damage in a numerical plate and in a beam based on the measurement of the displacement and acceleration.

An inverse use of the metamodel is also possible in order to find back the parameters that lead to a result of a simulation. The use of metamodels to solve an inverse problem has been implemented by Zhang [14] to find the characteristics of a damage from the measurement of eigenfrequencies. This numerical and experimental study shows that the use of a metamodel combined with a genetic algorithm obtains better results than a neural network to identify the parameters of a damage. Joy [8] has also shown that the use of a metamodel for inverse problem resolution is more efficient than a direct neural network.

In this paper, a numerical model of a simple aeronautic structure with one damage is proposed. The parameters describing the position $\left(x_{d a m}, y_{d a m}\right)$ of the dommage are used to generate a database with different damage configuration. Then a metamodel based on Radial Basis Function (RBF) interpolation is build in order to strongly reduce the computation time of the simulations for new damage positions. This surrogate model can be used to predict and generate a high quantity of data. It is then used to solve the inverse problem and find the position of a damage from SHM measurements. That lead to a new strategy for damage localisation.

\section{Numerical Model}

The finite element simulation is computed with the SDT software developed by SDTools.

The structure under study is a $300 \times 300 \times 2.4 \mathrm{~mm}$ plate in a composite material for the aeronautic industry. The plate has 8 plis [0 / 45 / 0 / 45 / 45 / 0 / 45 / 0]. The mechanical properties can be seen in the table 1 . The plate is modeled by shell elements with an linear elastic orthotropic behavior. 
The damage is modeled by a local cylindrical reduction of the all Young and Shear modulus across the plate thickness. The lower the ratio of the module it is, the more severe the damage. Here the diameter of the damage is fixed at $10 \mathrm{~mm}$ and the severity at $50 \%$.

The damping introduced in the model is stiffness proportional damping with a coefficient $\beta=1.5 \mathrm{e}-8$ for the mass term. Concerning the mechanical boundary conditions, free boundaries are chosen.

In order to monitor its structural health, the plate is equipped by 3 PZT. Each PZT can be used as actuator or sensor. The excitation signal is a burst with 5 cycles at $150 \mathrm{kHz}$ for the central frequency (figure 1).

The plate is meshed by a regular grid. The excitation frequency is fixed at 150 $\mathrm{kHz}$, which corresponds, according to the dispersion curves, to a wavelength $\lambda \approx$ $20 \mathrm{~mm}$ for this material. The dispersion curve for lamb wave can be seen figure 2 . The mesh size must be less than $10 \%$ of the wavelength, that is to say less than 2 $\mathrm{mm}$.

Table 1: Mechanical properties of the composite material

\begin{tabular}{lccccccc}
\hline Name & $E_{1}$ & $E_{2}$ & $v_{1}$ & $v_{2}$ & $\rho$ & $G_{1}$ & $G_{2}$ \\
\hline Value & 64.5 & 65.5 & 0.054 & 0.054 & 1570 & 4.8 & 4.8 \\
\hline Unit & $\mathrm{MPa}$ & $\mathrm{MPa}$ & - & - & $\mathrm{kg} / \mathrm{m3}$ & $\mathrm{MPa}$ & $\mathrm{MPa}$
\end{tabular}
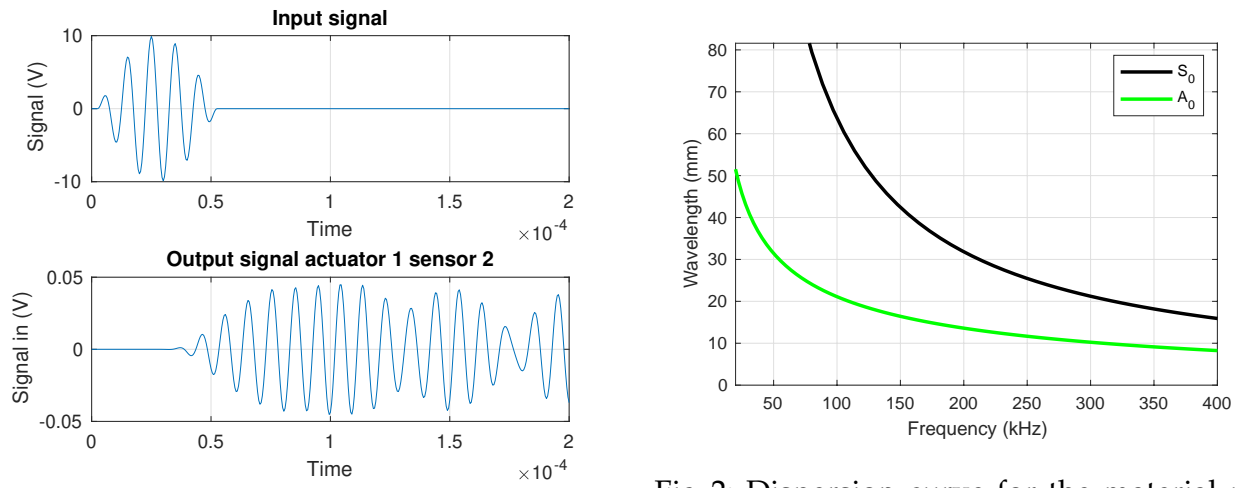

Fig. 1: Example of input and output signal

Fig. 2: Dispersion curve for the material under study 


\section{Metamodel}

A metamodel can be seen as a black box which for any input vector $\underline{x}$ associates a prediction $\underline{\hat{Y}}$, such as $\underline{\hat{Y}}=\mathcal{M}(\underline{x})$. The application $\mathcal{M}$ is computed during a training phase from a set of previously computed input and output. Let $p$ be the number of inputs parameters and $q$ the number of outputs. The set of $n$ inputs and outputs can be assembled respectively in a matrix $\underline{\underline{X}}$ and $\underline{\underline{Y}}$. The term $X_{j}^{i}$ corresponds to the value taken by the $j$-th parameter during the $i$-th simulation. The two matrices $\underline{X}, \underline{Y}$ contain the data used to train the metamodel. The data $\left(X_{j}, Y_{j}\right)$ associated to the $i$-th simulation are called a Snapshot or sampling point. The snapshots are computed beforehand from a numerical model, or measured from experimental data but can also be a mix of numerical and experimental data within the framework of a hybrid model [4].

$$
\underline{\underline{X}}=\left(\begin{array}{ccc}
X_{1}^{1} & \cdots & X_{1}^{n} \\
\vdots & \vdots & \vdots \\
X_{i}^{1} & \cdots & X_{i}^{n} \\
\vdots & \vdots & \vdots \\
X_{p}^{1} & \cdots & X_{p}^{n}
\end{array}\right)=\left(\begin{array}{llll}
x_{\operatorname{dam}_{1}} \cdots & x_{\text {dam }_{i}} \cdots & x_{\text {dam }_{n}} \\
y_{\text {dam }_{1}} \cdots & y_{d_{d a m_{i}}} \cdots & y_{d a m_{n}}
\end{array}\right) \text { and } \underline{\underline{Y}}=\left(\begin{array}{ccc}
Y_{1}^{1} & \cdots & Y_{1}^{n} \\
\vdots & \cdots & \vdots \\
Y_{i}^{1} & \cdots & Y_{i}^{n} \\
\vdots & \cdots & \vdots \\
Y_{q}^{1} & \cdots & Y_{q}^{n}
\end{array}\right)
$$

A surrogate model is build in order to predict a Lamb wave SHM control process (figure 3) based on the numerical model of the structure previously described. One damage is included on the structure. Two parameters $\left(x_{\text {dam }}, y_{\text {dam }}\right)$ describing the damage position are considered. The size and the severity of the damage are fixed. So the inputs are $\underline{X}=\left(x_{d a m}, y_{d a m}\right)$ with $x_{d a m}$ the horizontal position of the damage and $y_{\text {dam }}$ the vertical position of the damage. The $\underline{Y}$ output is the measured signal on a sensor for one actuator. Each way of actuator/sensor are considered independently : it is necessary to build one metamodel for each way of actuator/sensor.

\subsection{Sampling}

The construction of a metamodel requires beforehand the computation of a database $\underline{\underline{X}}, \underline{\underline{Y}}$ resulting from numerical simulations or experimental data. The choice of input variables $\underline{\underline{X}}$ for the training is not trivial because it strongly influences the precision and the speed of the metamodel. This choice of parameters $\underline{\underline{X}}$ is called Design Of Experiment, or Sampling within the framework of metamodels. Many DOE exist: random sampling, Latin hypercube Sampling [10], full grid sampling, scattered grid sampling, etc. In this work, the sampling strategy is based on a randomly perturbed regular grid [1] and can be seen figure 4.196 damage configurations are computed with 3 different actuators, so a total of 588 simulations. 


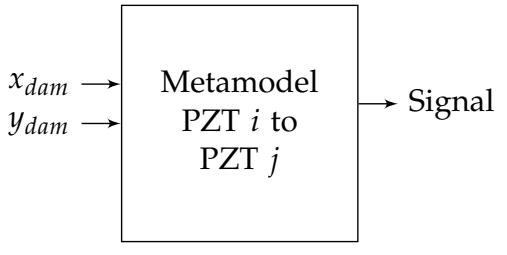

Fig. 3: Input/Output of the metamodel for actuator $i$ to sensor $j$

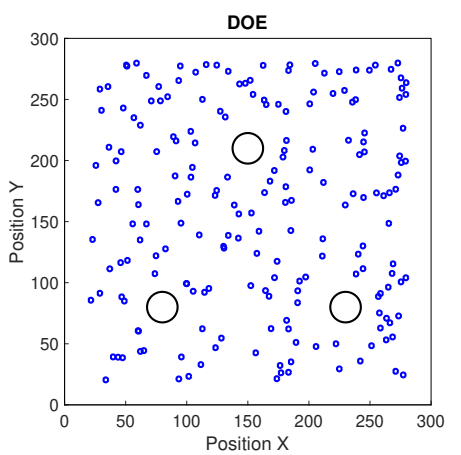

Fig. 4: DOE for plate structure

\subsection{Signal Compression}

The choice of the data representation is significant to obtain an efficient metamodel but also to facilitate its training. The data representation must be both parsimonious and, ideally, also have a physical meaning. The question is in which format express the output matrix $\underline{\underline{Y}}$ of the metamodel. The Singular Value Decomposition offers the possibility to compress data sparsingly and easily.

The SVD gives a base in which projecting the output signal. The basis is truncated according to the following criteria :

$$
\frac{\sum_{i=1}^{n_{r}} \lambda_{i}}{\sum_{i=1}^{n} \lambda_{i}} \geq 98 \%
$$

where $n_{S}$ is the number of snapshots, $n_{r}$ the number of modes in the reduced basis and $\left\{\lambda_{i}\right\}_{1}^{n}$ are the eigenvalue [6]. For this criteria, the reduced basis counts 16 vectors (figure 5 ).

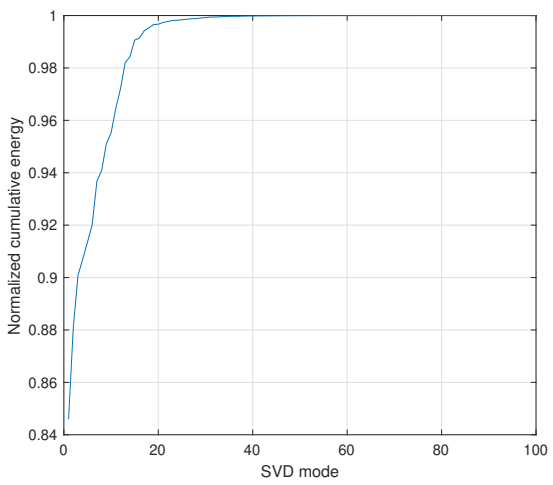

\subsection{Radial Basis Function Interpolation}

Fig. 5: Cumulative energy of SVD

Different metamodeling methods have been tested and evaluated. The method that got the best results is the Radial Basis Function interpolation (RBF). RBF were deployed by Hardy [7] for the interpolation of multi-variate scattered data. Radial basis functions have the particularity to decrease monotonically with distance from a central point. Many radial basis functions exist. In that section, it is considered that 
the metamodel is build only for one output $(q=1)$. For multiple outputs metamodels, each input is treated independently. The RBF model computes a linear combination of radial basis function $\varphi$ centered on each $\underline{X_{i}}$ sampling point. Each center $\underline{X_{i}}$ is associated with a weight $\omega_{i}$ such that :

$$
\forall x \in \mathbb{R}^{p}, \underline{\hat{Y}}(\underline{x})=\sum_{j=1}^{n} \omega_{j} \varphi\left(\left\|\underline{x}-\underline{X_{j}}\right\|\right)
$$

The coefficients $\omega_{i}$ can be computed by applying the interpolation condition :

$$
\forall i \in[1: n], Y_{i}=\sum_{j=1}^{n} \omega_{j} \varphi\left(\left\|\underline{X_{i}}-\underline{X_{j}}\right\|\right)
$$

resulting a linear system of equations:

$$
\underline{Y}=\underline{\underline{\varphi}} \underline{\omega}
$$

where $\underline{Y}$ is the vector of values of the function at the sampling points, $\underline{\omega}$ is the vector of weights of the radial basis function, and the matrix $\underline{\underline{\varphi}}$ is also known as the Gram matrix:

$$
\underline{\underline{\varphi}}=\left(\begin{array}{ccc}
\varphi\left(\left\|\underline{X_{1}}-\underline{X_{1}}\right\|\right) & \cdots \varphi\left(\left\|\underline{X_{n}}-\underline{X_{1}}\right\|\right) \\
\vdots & \ddots & \vdots \\
\left.\varphi\left(\left\|\underline{X_{1}}-\underline{X_{n}}\right\|\right)\right) & \cdots & \varphi\left(\left\|\underline{X_{n}}-\underline{X_{n}}\right\|\right)
\end{array}\right)
$$

The weights $\underline{\omega}$ are then computing by inverting the matrix $\underline{\varphi}$ or using a $\mathbf{Q R}$ decomposition for large matrices. The main parameters for RBF metamodels are the choice of the kernel function and the value of the kernel width defined by $\sigma$. In the

presented results, an Inverse MultiQuadric kernel function $\varphi(r, \sigma)=\frac{1}{\sqrt{1+\frac{r}{\sigma}}}$ is used with a width of 0.06 .

\subsection{Metamodel Evaluation}

Of the 196 snapshots from the simulations, $75 \%$ are used for training and $25 \%$ are kept for validation. The error between the predicted signal $\underline{\hat{Y}}$ and the validation signal $\underline{\hat{Y}}$ is computed with a FFT based implementation of the maximum of the correlation. This error is also a Damage Index used for damage detection.

$$
\text { Error }=1-\max \left(\frac{\operatorname{IFFT}(\operatorname{FFT}(\hat{\underline{Y}}) \overline{\operatorname{FFT}(\underline{Y})})}{\sqrt{E_{\underline{\hat{Y}}} E_{\underline{Y}}}}\right)
$$


Here the FFT is the Fast Fourrier Transform, IFFT the Inverse Fast Fourier Transform, $\mathrm{E}$ the mean value, $\underline{\hat{Y}}$ the predicted signal and $Y$ the reference signal. The results obtained by this surrogate model are presented in the figures 6-9. Figures 6 and 7 compare the predicted and simulated signals with minimum and maximum error respectively at the sensor 2 with the actuator 1 . There is a good match between the signals, indicating that the metamodel correctly predicts the effect of a damage. The figure 9 shows the evolution of the error with the distance to the nearest training point.

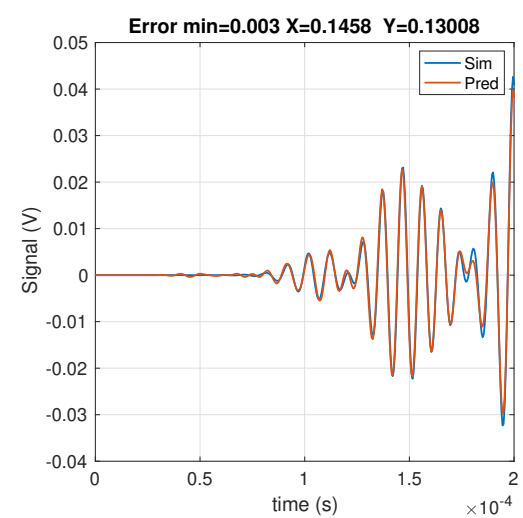

Fig. 6: Comparison of predicted/simulated Fig. 7: Comparison of predicted/simulated signal with minimum error for actuator 1 to sensor 2

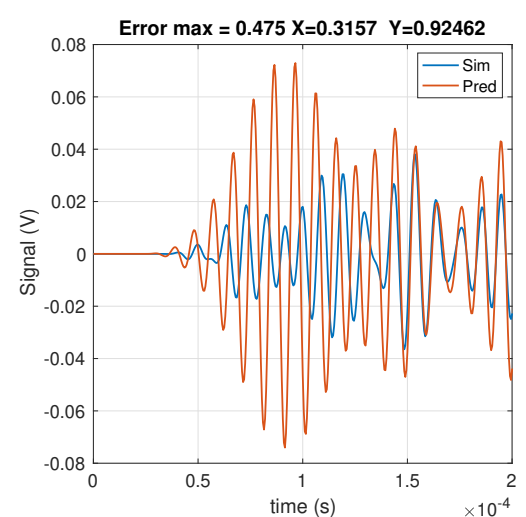

signal with maximum error for actuator 1 to sensor 2

\section{Inverse Problem resolution}

Metamodels allow to predict very quickly the results of a long simulation. Thus, a surrogate models can be used to predict signals on a large number of damage configurations. An inverse use of the metamodel is possible in order to find back the parameters that lead to a result of a simulation with the help of an optimisation algorithm. The surrogate model is used to find the $\left(x_{d a m}, y_{d a m}\right)$ position of a damage from a set of simulated signals. The signals come from the validation database, they have not been used to train the metamodel. A swarm particle minimization agorithm [9] seeks to minimize the global error. The global error here is the sum of the cross correlation based error (equation 6) for each actuator/sensor configuration between the signals predicted by the metamodel and the signals to be found 


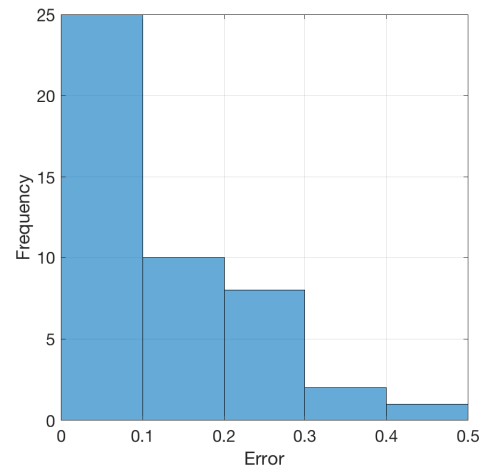

Fig. 8: Error histogram for actuator 1 to sensor

(equation 7).

$$
\text { Global Error }=\sum_{i=1}^{3} \sum_{j \neq j, j=1}^{3} \text { Error }_{i, j}
$$

where Error $_{i, j}$ is the error between the predicted and simulated signal at the sensor $j$ with the actuator $i$. The optimisation process can be seen figure 14. This method has been tested on 30 points that don't belong to the training set. The results are shown in figures 10-13. In the figure 10, the predicted position appears in red while the actual damage position is displayed in blue. The optimisation algorithm reached the convergence for all the test points. This method gives promising results but some points remain far from the actual position of the damage (points 1,2, 8, and 14). The reasons for these discrepancies are being investigated.

\section{Conclusion}

The previously described surrogate model allows to quickly predict the effect of a damage on the structure. It could be used to predict a large amount of data in order to evaluate statistically the SHM algorithms or to generate a database for other machine learning approach in SHM. The resolution of inverse problem based on a surrogate model gives promising results for damage localisation and must be tested with noisy and experimental data. Other parameters - such as the size, the severity, the thickness - need to be integrated into the damage model in order to gain realism. Moreother, the damage model used in this work is probably not the most realistic. 


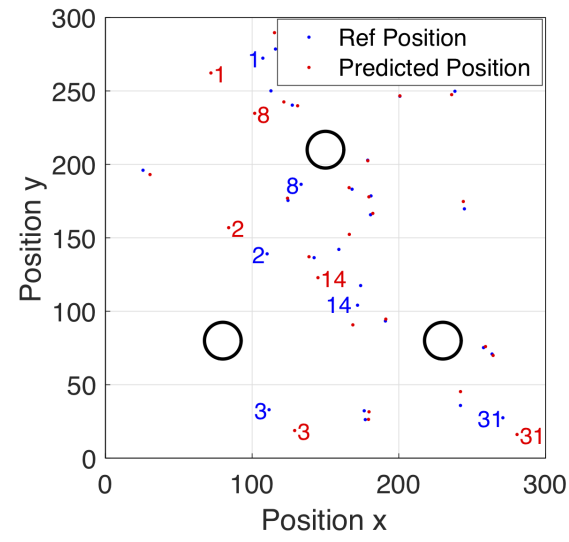

Fig. 10: Damage position estimation

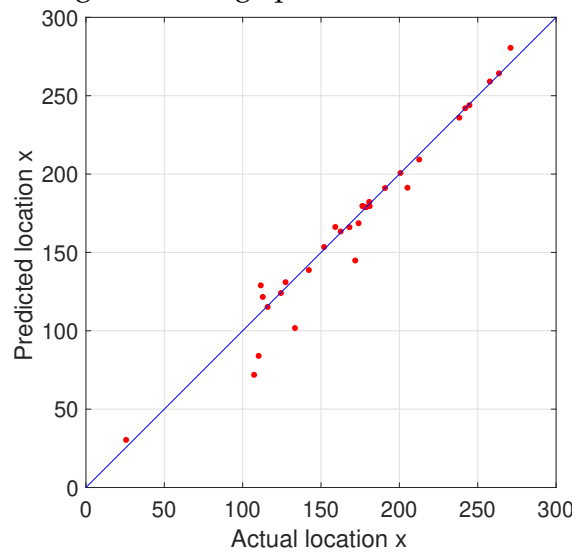

Fig. 11: Damage position $x$ estimation

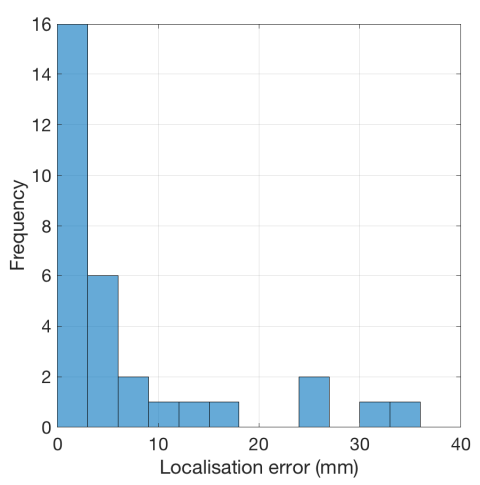

Fig. 12: Histogram of the error distance

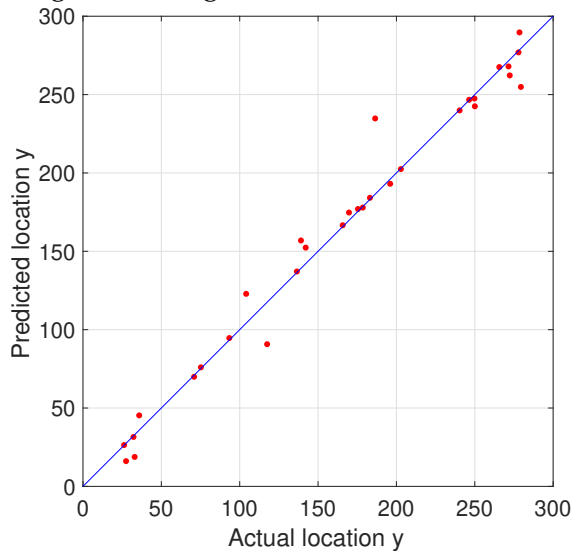

Fig. 13: Damage position y estimation

It will be necessary to study other damage models and fit numerical results with measurements carried out on a real plate. The inverse problem method should be extend to a metamodel with more parameters such as size, severity or shape of the dommage. That could lead to an efficient strategy to monitor structural health.

\section{Acknowledgement}

This work was supported buy the French Government via the program Fonds Unique Interministériel (project Monarque). 


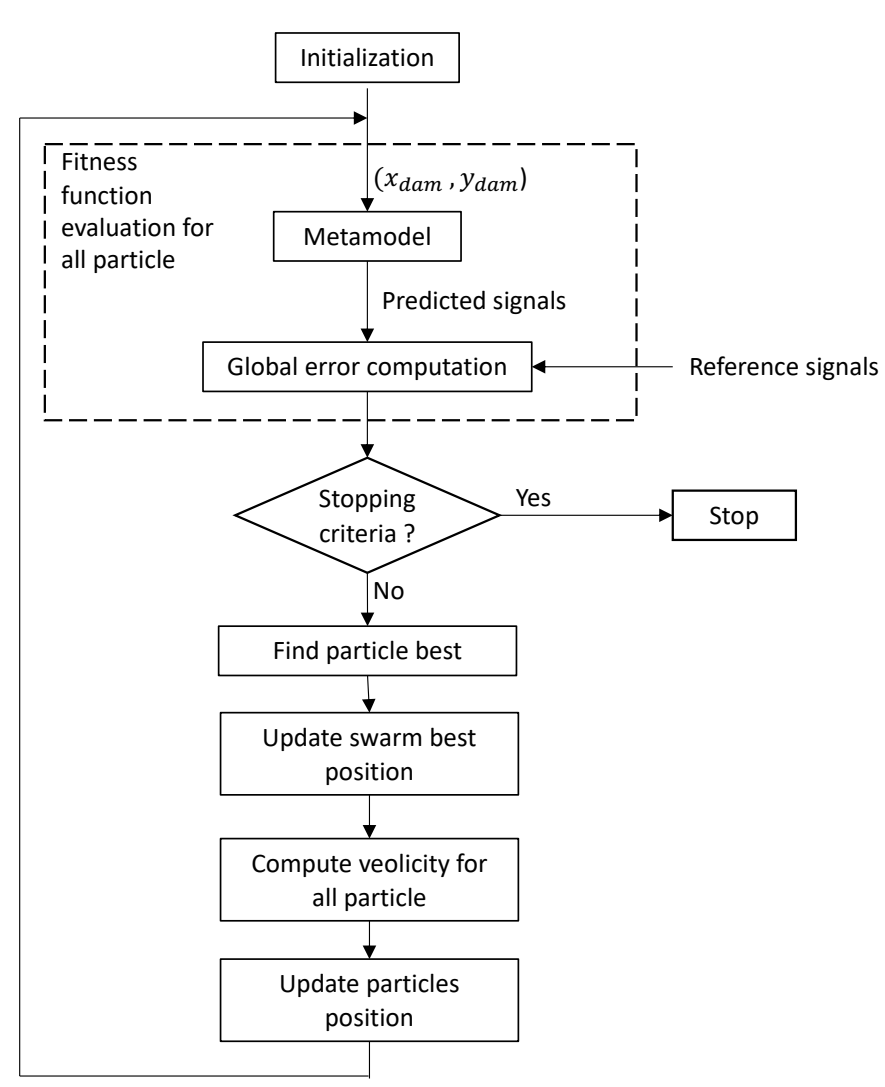

Fig. 14: Block Diagram for the inverse problem resolution with particle swarm optimisation

\section{References}

[1] Bachoc, F.: Asymptotic analysis of the role of spatial sampling for covariance parameter estimation of gaussian processes http://arxiv.org/abs/1301.4321

[2] Borate, P., Wang, G., Wang, Y.: Data-driven structural health monitoring approach using guided lamb wave responses 33(4), 04020033. https://doi.org/10.1061/(ASCE)AS.1943-5525.0001145, http://ascelibrary.org/doi/10.1061/\%28ASCE\%29AS.1943-5525.0001145

[3] Cawley, P.: Structural health monitoring: Closing the 
gap between research and industrial deployment 17(5), 1225-1244. https://doi.org/10.1177/1475921717750047, http://journals.sagepub.com/doi/10.1177/1475921717750047

[4] Chinesta, F., Cueto, E., Abisset-Chavanne, E., Duval, J.L., Khaldi, F.E.: Virtual, digital and hybrid twins: A new paradigm in data-based engineering and engineered data . https://doi.org/10.1007/s11831-018-9301-4, https://doi.org/10.1007/s11831-018-9301-4

[5] Giurgiutiu, V.: Structural health monitoring of aerospace composites. Elsevier

[6] Guo, M., Hesthaven, J.S.: Data-driven reduced order modeling for timedependent problems 345, 75-99. https://doi.org/10.1016/j.cma.2018.10.029, http://www.sciencedirect.com/science/article/pii/S0045782518305334

[7] Hardy, R.L.: Multiquadric equations of topography and other irregular surfaces 76(8), 1905-1915. https://doi.org/10.1029/JB076i008p01905, http://doi.wiley.com/10.1029/JB076i008p01905

[8] Joy, E.J., Menon, A.S., Biju, N.: Implementation of kriging surrogate models for delamination detection in composite structures 27(6), 096369351802700. https://doi.org/10.1177/096369351802700604, http://journals.sagepub.com/doi/10.1177/096369351802700604

[9] Kennedy, J., Eberhart, R.: Particle swarm optimization. In: Proceedings of ICNN'95 - International Conference on Neural Networks. vol. 4, pp. 1942-1948 vol.4 (1995)

[10] Kleijnen, J.P.: Kriging metamodeling in simulation: A review 192(3), 707-716. https://doi.org/10.1016/j.ejor.2007.10.013, https://linkinghub.elsevier.com/retrieve/pii/S0377221707010090

[11] Moriot, J., Quaegebeur, N., Le Duff, A., Masson, P.: A modelbased approach for statistical assessment of detection and localization performance of guided wave-based imaging techniques 17(6), 1460-1472. https://doi.org/10.1177/1475921717744679, http://journals.sagepub.com/doi/10.1177/1475921717744679

[12] Ostachowicz, W., Soman, R., Malinowski, P.: Optimization of sensor placement for structural health monitoring: a review 18(3), 963-988. https://doi.org/10.1177/1475921719825601, http://journals.sagepub.com/doi/10.1177/1475921719825601

[13] Schubert Kabban, C.M., Greenwell, B.M., DeSimio, M.P., Derriso, M.M.: The probability of detection for structural health monitoring systems: Repeated measures data 14(3), 252-264. https://doi.org/10.1177/1475921714566530, http://journals.sagepub.com/doi/10.1177/1475921714566530

[14] Zhang, Z., Pan, J., Luo, W., Ramakrishnan, K.R., Singh, H.K.: Vibration-based delamination detection in curved composite plates 119, 261-274. https://doi.org/10.1016/j.compositesa.2019.02.002, https://linkinghub.elsevier.com/retrieve/pii/S1359835X19300430 